\title{
Zur Kenntnis der Adsorption, I.
}

\section{Chemische Hysteresis der Stärke.}

\author{
Von Adam Rakowski (Moskau).
}

(Eingegangen am 18. Juni 1911)

Die Erscheinung der chemischen Hysteresis besteht darin, dab die Kurven der Wässerung und Entwässerung ein und desselben Präparats eines kolloiden Stoffes in "festem" $\mathrm{Zu}$ stande (Gel) nicht zusammenfallen. Die chemische Hysteresis ist von J.M. van Bemmelen bei den Hydroxyden des Kupfers und Eisens und bei der Kieselerde ') entdeckt worden. Wenn wir auf der Abszissenachse die Wassermenge (in Proz. oder Molen) im Gel, auf der Ordinate den Druck des Wasserdampfes im umgebenden Gasmittel eintragen, so erhalten wir für ein jedes Gelpräparat (der von J.M. van Bem melen untersuchten Substanzen) drei Kurven der Dampfspannung: die Kurve der primären Entwässerung, die Kurve der Wässerung und die Kurve der nachfolgenden Entwässerung. Auf jeder von diesen drei Kurven ist die Bewegung nur in einer Richtung möglich; der Uebergang von einer Kurve auf die andere ist, abgesehen von zwei gemeinsamen Punkten -- den Spitzen des Zyklus - vermittels einer Reihe von Zwischenkurven möglich; die letzteren sind reversibel, und die Bewegung auf denselben kann nach beiden Richtungen erfolgen. Das Eisenhydroxyd stellt den allgemeinsten, vonJ.M.v a $\mathrm{n} B \mathrm{~m}$ m e le n untersuchten Fall dar, hier fallt die Kurve der Wässerung in ihrer ganzen Ausdehnung nicht mit der Kurve der Entwässerung zusammen; besonders charakteristisch für die Kurven der Entwässerung beider Hydrosole ist der fast horizontale Verlauf des mittleren Teils.

Die Erscheinungen der chemischen Hysteresis sind, soviel mir bekannt ist, auBer von J. M. van Bemmelen bis jetzt von keinem näher untersucht worden. Die Frage, ob die chemische Hysteresis auch dann gilt, sobald praktisch ein vollkommenes Gleichgewicht ${ }^{2}$ ) erreicht ist, ob die chemische Hysteresis eine

1) J. M. van Bemmelen, Die Absorption (Dresden 1910).

7) Das volle Gleichgewicht $z$ wischen dem Wasserdampf und dem Wassergehalt im Gel (der Kieselerde) wurde in den Versuchen von J.M. va $n$ Be inmelen außerst langsam (in $1-2$ Jahren) erreicht; er begnügte sich mit einem annahernden Gleichyewicht, indem er den Prozeß als beendet ansah, sobald der tägliche Wasserverlust im $\mathrm{SiO}_{\mathrm{g}}$ - Gel nicht $0,01 \mathrm{Mol}$. Wasser uberschritt, was bel $\mathrm{SiO}_{2} 2 \mathrm{H}_{2} \mathrm{O}$ ca. 0,18 Proz. ausmacht (J.M. van Bemmelen ib. 203). allgemeine oder ausschlieBliche Eigenschaft der Gele bildet, erfordert zu ihrer Lösung eine ganze Reihe von Versuchen mit verschiedenen kolloiden Körpern; ich habe mir vorgenommen, die organischen Kolloide zu bearbeiten and habe zunăchst fünf verschiedene Stärkearten untersucht; die Versuche mit drei von diesen - namlich der Weizenstärke, dem Arrowroot St. Vinzent und der Kartoffelstärke - sind abgeschlossen, und die Resultate werden in vorliegender $\mathbf{A b}$ handlung mitgeteilt. Die Versuche mit Reisstärke und mit dem Bermuda-Arrowroot werden noch fortgesetzt, um einige spezielle Fragen hinsichtlich des Charakters der Zwischenkurven zu Iosen; die betreffenden Resultate werden in nächster Zeit veröffentlicht werden.

Die Stärke wurde einer sorgfaltigen Reinigung mit Wasser, Alkohol, Aether und wieder mit Alkohol und Wasser unterworfen. Der Aschegehalt in allen Stärkearten betrug weniger als 0,1 Proz., von Stickstoff sind in der Kartoffelstärke und dem Arrowroot nur Spuren (bedeutend weniger als 0,1 Proz.), in der Reisund Weizenstärke um 0,1 Proz.

Zur Erreichung eines abgeschlossenen Raumes mit einem bestimmten Wasserdampfdruck bediente ich mich des Exsikkators, der mit ca. $400 \mathrm{ccm} \mathrm{H}_{2} \mathrm{SO}_{4}$-Lösung gefüllt wurde. Nach den Daten von Regnaulis) berechnete ich die Zusammensetzung der Lösung, die die gewünschte Dampfspannung ergeben muBte, nach den Tabellen von Domke fand ich das entsprechende spezifische Gewicht; das letztere wurde zuerst durch Aräometer und schlieblich durch Pyknometer mit einer Genauigkeit bis zur vierten Dezimalstelle bestimmt. Von Zeit zu Zeit wurde das spezifische Gewicht kontrolliert $\left.{ }^{5}\right)$. Um einen mit Wasserdampf ge-

b) Landolt-Bornstein, Physikalisch-chemische Tabellen (3. Aull. 1905), S. 166 .

4) Desgl., S. 326.

5) Die Daten von Regnault schließen mit einer 24 proz. $\mathrm{H}_{2} \mathrm{SO}_{4}$-Losung, zur Bestimmung der Dampfspannung einer ca. 10 proz. $\mathrm{H}_{2} \mathrm{SO}_{4}$-Losung mus man schon seine Zuflucht zur Interpolation nehmen oder zur graphischen Methode ein Gebiet, wo die Spannungskurve eine verhalitnisma日ig scharfe Krümmung bildet, weshalb beide Arten der Berechnung ungenau sind und verschiedene Werte crgeben; der Unterschied zwischen den durch Interpolation und auf graphischem Wege erhaltenen Werte errelcht $0,5 \mathrm{~mm}$. Ich habe 
sättigten Raum zu erhalten, benutzte ich statt Wasser eine einprozentige $\mathrm{H}_{2} \mathrm{SO}_{4}$-Lösung; die Abnahme der Dampfspannung ist in diesem Falle sehr gering, und man kann annehmen, daB man einen praktisch mit Wasserdampf gesätligten Raum hat ${ }^{6}$ ).

Der Verlauf der Arbeit war folgender: Die Starke wurde, mit Wasser übergossen, nach einer Stunde dasselbe abgesaugt, die nasse Stärke zwischen Filtrierpapier abgeprebt und darauf in einem Mörser zerrieben, um das "mechanisch" anhaftende Wasser ${ }^{7}$ ) zu verteilen; einen Teil der Stärke $(2-10 \mathrm{~g}$ bei verschiedenen Versuchen) brachte ich auf einem Gläschen in einen Exsikkator mit einer einprozentigen $\mathrm{H}_{2} \mathrm{SO}_{4}$ Lösung - der übrige Teil desselben Präparats diente zur Wasserbestimmung ${ }^{8}$ ). Da die Versuche ohne Unterbrechung im Laufe von einigen Jahren in mehreren Exsikkatoren geführt wurden, so konnte von keinem Thermostat die Rede sein. Die Exsikkatoren wurden in einem Schrank untergebracht und die Zimmertemperatur gemessen, im Durchschnitt betrug dieselbe $19^{\circ}$. Im Laufe von 4 Jahren fiel sie nur einmal bis $15^{\circ}$ und ca. zweimal stieg sie auf $22^{\circ}$, im übrigen schwankte sie zwischen 18 und $20^{\circ}$. Wir werden weiter sehen, $\mathrm{daB}$ die Temperatur praktisch von keinem Einflutb anf die Resultate derartiger Versuche ist. Schon die ersten Versuche zeigten mir, dab man bei der Stärke ein praktisch vollständiges Gleichgewicht nach $I$ bis 2 Monaten (bei $19^{\circ}$ ) erreicht. Häufig war es nicht möglich, ein konstantes Gewicht zu erhalten, die Schwankungen betrugen $3-5 \mathrm{mg}$. In den meisten Fallen hielt ich die Stärke im Exsikkator, bis das Gewicht im Laufe von mehreren $(5-7)$ Tagen sich nicht änderte oder die Aenderung (in 3-4 Tagen) 0,01 Proz. nicht überstieg. Das beobachtete Gleichgewicht in der Stärke ist praktisch vollständig, es ist aber nicht bewiesen, daß bei denselben Bedingungen

mich des auf graphischem Wege erhaltenen Wertes bedient (11,4 proz. $\mathrm{H}_{2} \mathrm{SO}_{4}$-Losung, $15,5 \mathrm{~mm}$ Druck). Mit Ausnahme dieser Losung geben alle übrigen graphisch sowohl als auch durch Interpolation vollkommen ubereinstimmende Werte (der Unterschied ist gewohnlich geringer als $0,1 \mathrm{~mm}$ ).

- Landolt-Börnstein, desgl. S. 167

7) Ausgehend von der Theorie (der Thermodynamik irreversibler Prozesse) von $P$. Duhem könnte man annehmen, daB im gegebenen Falle wir es nicht mit (buchstäblich) .mechanisch" anhaftendem Wasser $z u$ tun haben. Auf diese Frage komme ich noch in einer späteren Abhandlung zurück.

8) Nach Beendigung der Versuche wurde die Menge der absolut trockenen Stärke direkt auf dem Glaschen bestimmt. der ProzeB nicht äuBerst langsam Jahre hindurch dauert.

Sobald die Stärke im Exsikkator Nr. 1 über einer einprozentigen $\mathrm{H}_{2} \mathrm{SO}_{4}$-Lösung ein konstantes Gewicht erreichte, brachte ich sie in den Exsikkator Nr. 2 mit einem Wasserdampfdruck von $15,5 \mathrm{~mm}^{9}$ ), und aus dem letzteren der Reihe nach in Exsikkatoren mit einem Druck von $12,65 \rightarrow 11,0 \rightarrow 9,0 \rightarrow 6,95 \rightarrow 5,07 \rightarrow 3,0 \rightarrow 2,0$ $\longrightarrow 0,7 \rightarrow 0,0 \mathrm{~mm}$. War die Stärke schlieblich über der $\mathrm{H}_{2} \mathrm{SO}_{4}$ vom spezifischen Gewicht 1,84 $(0,0 \mathrm{~mm})$ entwässert, so brachte ich dieselbe der Reihe nach in Exsikkatoren mit zunehmendem Druck bis zum ersten, darauf wieder in umgekehrter Reihenfolge und endlich zum vierten Mal bis zum ersten Exsikkator; im Durchschnitt passierte also ein jedes Präparat viermal die Exsikkatoren, in seltenen Fällen fünfmal und zur Lösung spezieller Fragen zweimal.

Für die Weizenstärke habe ich folgende in der Tabelle I angeführten Werte erhalten und die Resultate in Fig. 1 graphisch dargestellt.

Tabelle I

Weizenstärke.

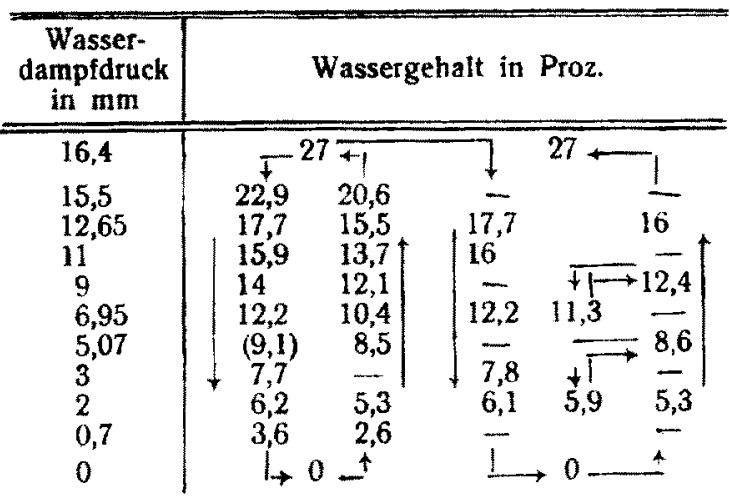

In Vergleich mit den Kurven von J. M. van Bemmelen sind die yon mir erhaltenen einfacher (ohne eine horizontale Mitte). Die Divergenz der Kurven ist bedeutend geringer, als bei den anorganischen Kolloiden - bei der Weizenstärke beträgt dieselbe $2-3$ Proz.; die Fehler beim Experiment übersteigen, meiner Meinung nach, nicht 0,5 Proz. Vergleicht man die beiden Kolumnen ( $t)$ und die beiden $(t)$, so sehen wir, daß die Divergenz der Kurven der Wässerung und Entwässerung nicht durch die Aenderung der Stärke mit der Zeit erklärt werden kann. Zwischen dem Erhalten der entsprechenden Zahlen zweier syn-

9) Dieser Wert ist ungenau; vgl. früher. 


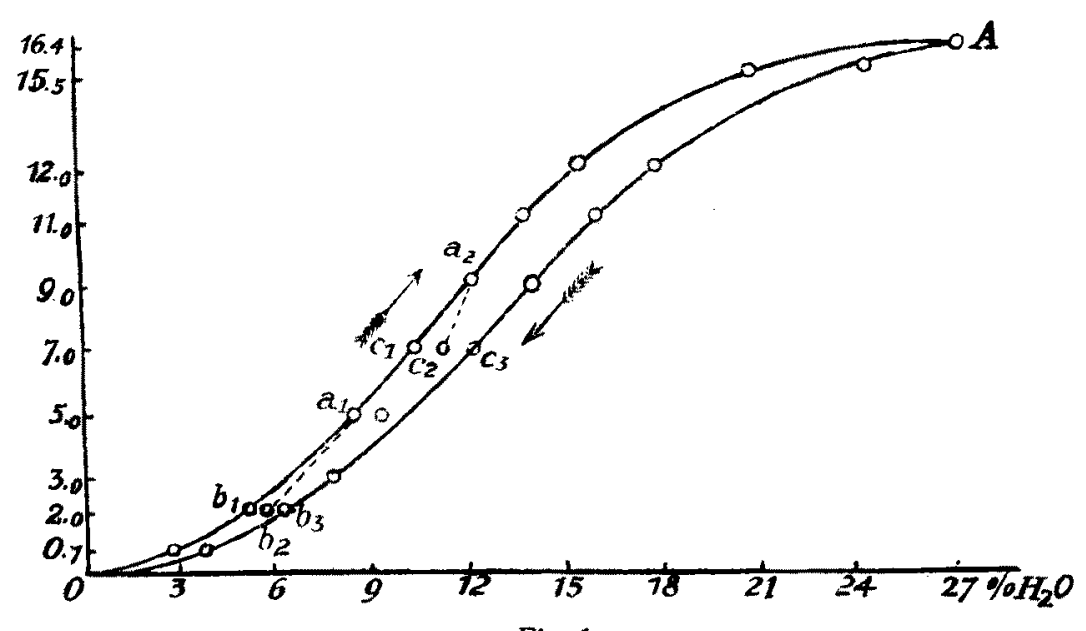

Fig. 1

onymer Kolumnen sind ca. 2 Jahre vergangen, wir finden aber eine vollkommene Uebereinstimmung zwischen den beiden Kolumnenpaaren.

Die Kurven der Wässerung und Entwăsserung fallen nur in zwei Punkten zusammen, welche dem Null- und dem Maximaldruck entsprechen; eine jede der Kurven ist irreversibel; die $Z$ wischenkurven, $z$. B. a $b_{2}, a_{2} c_{2}$, sind reversibel.

Tabelle II

Arrowroot St. Vinzent.

\begin{tabular}{|c|c|c|c|}
\hline $\mathbf{m m}$ & \multicolumn{3}{|c|}{ Wassergehalt in Proz. } \\
\hline 16,4 & \multicolumn{3}{|c|}{ T 28,5} \\
\hline 15,5 & 24,2 & $(24,5)$ & \\
\hline 12,65 & 18,9 & 16,6 & 18,6 \\
\hline 11 & 17 & 14,6 & 16,7 \\
\hline 9 & 15,1 & 13 & $\overline{12}$ \\
\hline 6,95 & & 11,1 & 13 \\
\hline 5,07 & & 9,1 & - \\
\hline $\begin{array}{l}3 \\
2\end{array}$ & $\begin{array}{l}+8,5 \\
6,7\end{array}$ & $\begin{array}{l}7 \\
5,7\end{array}$ & $\overline{6,8}$ \\
\hline 0,7 & 3,3 & 2,8 & - \\
\hline 0 & L & 5 & 0 \\
\hline
\end{tabular}

Die Kurven des Arrowroot St.Vinzent sind vollkommen analog den Kurven der Weizenstärke. Für die Kartoffelstärke (Präparat Nr. I) habe ich ein komplizierteres Bild gefunden. Beim anfänglichen Entwasssern habe ich den Wassergehalt nicht bis auf 0 gebracht, sondern bedeutend früher die Stärke in den Exsikkatoren weiter nach oben gebracht, und wiederum beim höchsten Punkt habe ich nicht eine vollständige Sättigung der Stärke mit Wasser (33 Proz.) abgewartet, sondern dieselbe weiter nach unten zu in die Exsikkatoren gebracht. Beim abermaligen Entwässern und Wässern habe ich mich überzeugt, daB auch die Kartoffelstärke vollkommen entwässert werden kann, und daB beide Kurven einen gemeinsamen höchsten Punkt haben. Allem Anschein nach hat sich jene Tatsache, daß ich beim anfänglichen Entwässern und Wässern nicht ein vollständiges Gleichgewicht in den auBersten Punkten erreicht habe, im Nichtübereinstimmen der beiden synonymen Kolumnen + und + (Fig. 2) geäuBert.

Beim gegebenen Präparat der Kartoffelstärke wurden drei $Z$ wischenkurven b c erhalten, welche vollkommen analog den Kurven der Weizenstärke waren. Ich habe noch zwei Präparate der Kartoffelstärke untersucht, welche zu verschiedenen Zeiten und aus verschiedenen Gegenden akquiriert worden waren. Das Präparat II, welches im ersten Exsikkator (Wasser) ein Gleichgewicht erreicht hatte, enthielt im Exsikkator mit einem Druck von $6,95 \mathrm{~mm}$ 14,72 Proz. Wasser, bei gleichem Druck enthal-

Tabelle III

Kartoffelstärke.

\begin{tabular}{|c|c|c|c|c|}
\hline $\mathrm{mm}$ & \multicolumn{4}{|c|}{ Wassergehalt in Proz. } \\
\hline $\begin{array}{l}16,4 \\
15,5 \\
12,65 \\
11 \\
9 \\
6,95 \\
5,07 \\
3 \\
2 \\
0,7 \\
0\end{array}$ & $\begin{array}{l}33 \\
29,1 \\
22,05 \\
19,75 \\
17,5 \\
15 \\
12,2 \\
9,1 \\
7 \\
3,2 \\
L \\
\end{array}$ & $\frac{30,4}{\frac{18,4}{13,8}} \frac{\frac{9,4}{5,7}}{\frac{5,7}{4}}$ & $\frac{1}{21,3}$ & $\underset{15}{15}=\frac{33}{17,9}$ \\
\hline
\end{tabular}




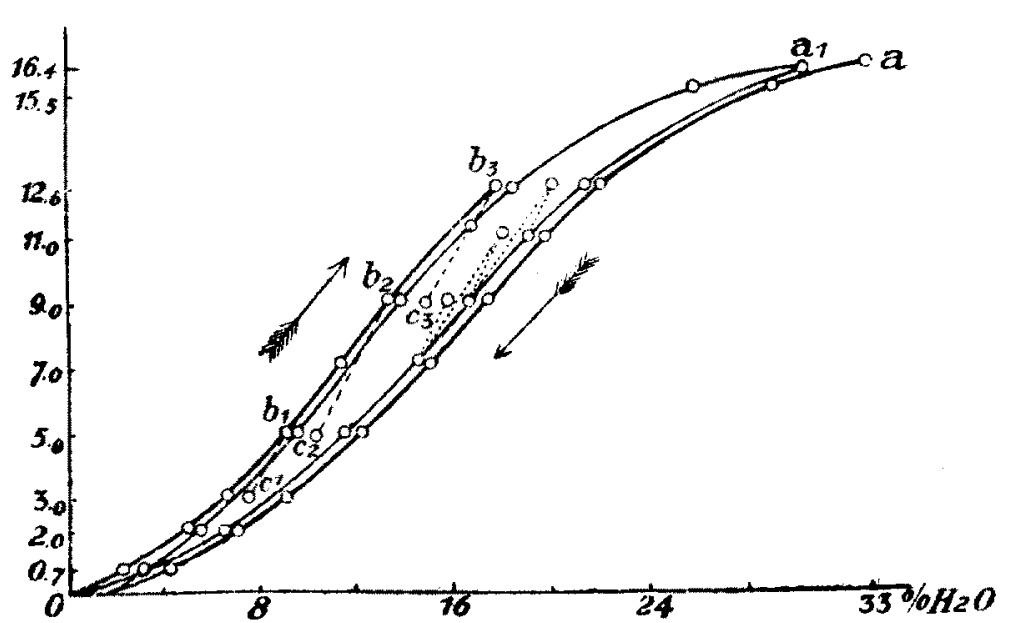

Fig. 2

hielt das Präparat I 15 Proz., das Prăparat III 14,5 Proz. Wasser. Nach dem Trocknen über $\mathrm{H}_{2} \mathrm{SO}_{4}$ vom spezifischen Gewicht 1,84 waren im Präparat II im Exsikkator mit einem Druck von 6,95 mm 11,35 Proz. Wasser. Beide Punkte liegen zwischen zwei synonymen Kurven, welche für das Präparat I erhalten wurden.

Zur Lösung der Frage, ob die Kurven + und + tatsächlich im höchsten Punkt zusammentreffen, wurden von mir besondere Versuche (Präparate I und III) angestellt, wobei ich folgende Resultate erhielt:

1. Feuchte Stärke I enthielt über einer einprozentigen $\mathrm{H}_{2} \mathrm{SO}_{4}$-Lösung nach Erreichung des Gleichgewichts 32,6 Proz. Wasser; 2. feuchte Stärke III 33 Proz.; 3. lufttrockene Stärke III 33 Proz.; 4. absolut trockene Stärke III 32,85 Proz.; 5. absolut trockene Stärke 1 32,9 Proz.

Das Präparat III der Kartoffelstărke brachte ich abwechselnd in einen Exsikkator mit höchstem und mit niedrigstem Dampfdruck. Die erhal- tenen Daten sind durch die punktierten Kurven in Fig. 2 dargestellt; sie beweisen nochmals die Irreversibilität der Hauptkurven und die Existenz der Hysteresis.

Nachdem das Präparat $l$ der Kartoffelstärke viermal die Exsikkatoren passiert hatte, trocknete ich dasselbe von neuem und hielt es im Laufe von 6 Stunden bei $100^{\circ}$; nach dem Abkühlen brachte ich es in einigen Exsikkatoren nach oben und unten, darauf unterwarf ich es 6 Stunden lang einer Temperatur von $120^{\circ}$, brachte es wieder in die Exsikkatoren, erwärmte es von neuem auf $137^{\circ}$ (5 Stunden lang) und lief es durch die Exsikkatoren passieren (Tabelle IV, Fig. 3).

Für einige Kurven gibt es nur vier Punkte, aber auch diese vier Punkte genügen, um die Lage der Kurve zu bestimmen. Es war unmöglich, die Stărke durch eine gröBere Anzahl von Exsikkatoren zu führen, da allein ca. 3 Jahre notwendig waren, um nur die Daten der Tabelle IV zu erhalten. Wie man aus Fig. 3 ersieht, verschiebt das vorhergehende Erwärmen der Stărke alle Kurven nach links, wobei eine besonders starke Verschiebung der Temperaturen wahrgenommen wird, welche höher als $100^{\circ}$ sind. Die Kurve der Wässerung (punktiert) der vorher auf $137^{\circ}$ erwärmten Stärke zeigt eine neue $\mathrm{Er}$ scheinung - sie ist im Verhältnis zur Kurve der Wässerung der auf $120^{\circ}$ erwärmten Stärke nach rechts verschoben. Die letzte Kurve auf Fig. 3 erscheint als äuBerste Grenze der Verschiebung der Kurven. Aus den Versuchen von Solomon ${ }^{10}$ ) wissen wir, daB $120^{\circ}$

Tabelle IV

Kartoffelstärke. Der Einfluß eines vorhergehenden Erwărmens. Die Temperatur in den Exsikkatoren beträgt $19^{\circ}$.

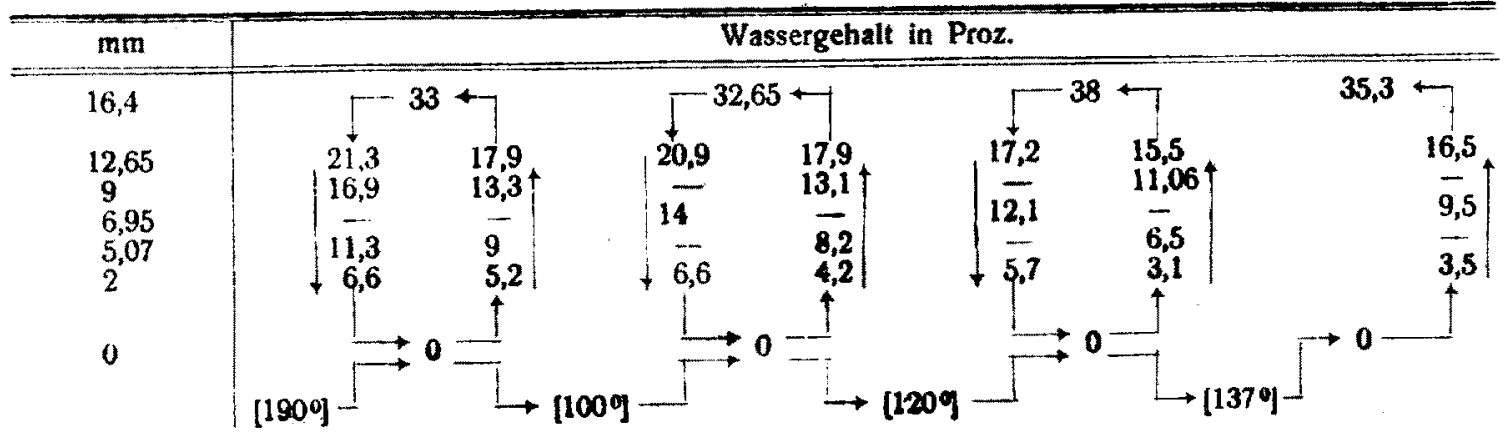




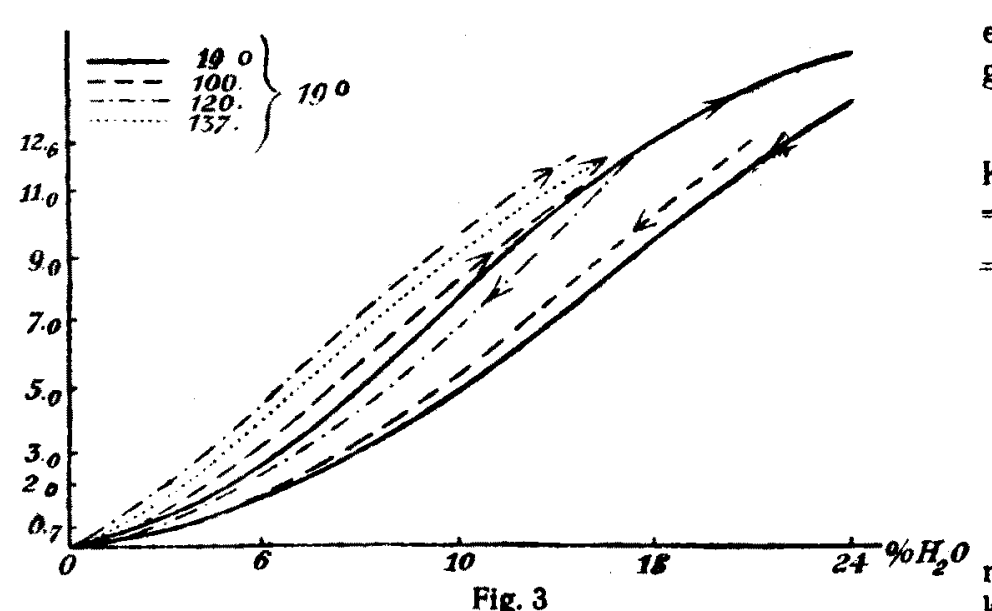

erhielt ich doch im Resultat eine gleichmäßige Kurve.

Tabelle V

Kartoffelstärke. Temperatur $30^{\circ}$

\begin{tabular}{c|cc}
\hline $\mathrm{mm}$ & Wassergehalt in Proz. \\
\hline 24,25 & 21,9 & \\
17,75 & 17,2 & \\
14 & 14,5 & \\
9,9 & 12 & 9,25 \\
6 & $+8,9$ & 7 \\
4 & 6,75 & 5,6 \\
0 & & 0
\end{tabular}

Das Gewicht des Gläschens mit der Stärke, welche im Exsikkator bei $30^{\circ}$ mit einem Druck einen Grenzwert der Temperatur bildet, bei welcher die Stärke, ohne sich sichtbar zu verändern, alles Wasser zu verlieren imstande ist.

Bei allen geschilderten Experimenten betrug die Temperatur in den Exsikkatoren 19 ${ }^{\circ}$. Trotz der fortwährenden Temperaturschwankungen bis zu $2^{\circ}$, dazwischen auch noch größerer, ergaben die Punkte gleichmäBig verlaufende Kurven, ohne daß man die Schwankungen wahrnehmen konnte. Zur endgültigen Feststellung des Temperatureinflusses auf das Gleichgewicht zwischen Stärke und Wasser führte ich die Kartoffelstärke II durch mehrere Exsikkatoren in beiden Richtungen bei einer Temperatur von $30^{\circ}$ (in den Exsikkatoren). Zu diesem Zweck bediente ich mich einer Abteilung des Laboratoriumkellers, in welcher sich der Kessel für die Wasserheizung befand. Obgleich hier die Temperaturschwankungen bedeutend waren,

19) Sol o m o n, Journ. f. prakt. Chem. 28, 90 (1883). Hier ist eine Methode angegeben, den Wassergehalt der Stăke $z \mathfrak{u}$ bestimmen. von $9,9 \mathrm{~mm}$ das Gleichgewicht erreicht hatte, betrug 18,012 g; dieser Exsikkator wurde mit der Stărke in einen Raum von $19^{\circ}$ gebracht, und nach zwei Monaten wog das Gläschen mit der Stärke $18,013 \mathrm{~g}$. Die Temperatur hat also ohne Zweifel keinen EinfluB auf das Gleichgewicht eines derartig komplizierten Systems, wie die wässerige Stärke und die durch ein Gasmittel von ihm getrennte Schwefelsalurelösung, mit anderen Worten - die Temperaturkoeffizienten der Dampfspannung des Wassers in der Stärke und des Wassers in den Schwefelsäurelösungen kommen einander schr nahe. Um diese Annahme zu beweisen, habe ich folgende Berechnung'gemacht. Da das Präparat II der Kartoffelstärke, für welches die Daten bei $30^{\circ}$ erbalten wurden, bei $19^{\circ}$ in einigen $\mathrm{Ex}$ sikkatoren Punkte ergab, welche zwischen zwei synonymen Kolumnen der Tabelle Ill lagen, so nahm ich für die Berechnung das Mittel dieser beiden Kolumnen:

Tabelle VI

\begin{tabular}{|c|c|c|c|c|c|c|c|}
\hline $\begin{array}{c}\text { Dampi- } \\
\text { spannung } \\
\mathrm{mm} \\
\end{array}$ & $\begin{array}{c}\text { Wassergehalt } \\
\text { in der Stärke } \\
\mathbf{m}\end{array}$ & $\begin{array}{c}\text { in Proz. bei } 190 \\
\text { in der } \mathrm{H}_{2} \mathrm{SO}_{4} \\
\text { Losung } \\
n\end{array}$ & $\begin{array}{l}\text { Spannum } \\
\text { der Stärke } \\
(\mathrm{m})\end{array}$ & $\begin{array}{c}\text { bei } 30^{\circ} \\
\text { der } \mathrm{H}_{2} \mathrm{SO}_{4} \\
\text { Losung } \\
(\mathrm{n})\end{array}$ & $\begin{array}{c}\text { Differenz } \\
\text { zwischen } \\
\text { den Rubriken } \\
4 \text { und } 5\end{array}$ & $\begin{array}{l}\mathbf{n} \\
\mathbf{m}\end{array}$ & $\frac{n-15,4}{m}$ \\
\hline $\begin{array}{l}2 \\
3 \\
4 \\
5 \\
6 \\
7 \\
8 \\
9 \\
10 \\
11 \\
12 \\
12,65 \\
16,4\end{array}$ & $\begin{array}{l}6,8 \\
8,4 \\
10 \\
11,6 \\
13 \\
14,4 \\
15,8 \\
17,2 \\
18,4 \\
19,7 \\
20,9 \\
21,7 \\
33\end{array}$ & $\begin{array}{l}34,8 \\
40 \\
43,9 \\
46,7 \\
49,7 \\
53 \\
56,3 \\
59,05 \\
61,8 \\
65 \\
68,7 \\
71,5 \\
100\end{array}$ & $\begin{array}{c}4,05 \\
5,5 \\
7,4 \\
9,4 \\
11,5 \\
13,8 \\
15,8 \\
17,75 \\
19,4 \\
21,2 \\
22,9 \\
24 \\
\ldots\end{array}$ & $\begin{array}{r}3,84 \\
5,95 \\
7,95 \\
9,88 \\
11,77 \\
13,72 \\
15,67 \\
17,46 \\
19,26 \\
21,09 \\
22,96 \\
24,22 \\
-\end{array}$ & $\begin{array}{r}+0,21 \\
-0,45 \\
-0,55 \\
-0,48 \\
-0,27 \\
+0,08 \\
+0,13 \\
+0,29 \\
+0,24 \\
+0,11 \\
-0,06 \\
-0,22 \\
-\end{array}$ & $\begin{array}{l}5,12 \\
4,76 \\
4,39 \\
4,03 \\
3,82 \\
3,68 \\
3,57 \\
3,43 \\
3,36 \\
3,30 \\
3,29 \\
3,30 \\
3,03\end{array}$ & $\begin{array}{l}2,85 \\
2,93 \\
2,85 \\
2,70 \\
2,64 \\
2,61 \\
2,59 \\
2,53 \\
2,52 \\
2,52 \\
2,55 \\
2,59 \\
2,56\end{array}$ \\
\hline
\end{tabular}


bei $12,65 \mathrm{~mm} 21,7$ Proz. Wasser

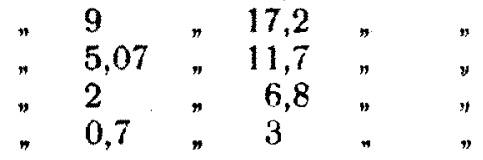

Aus diesen Daten wollen wir durch Interpolation den Wassergehalt in den Stärkearten berechnen, deren Dampfspannung $=2,3,4$ usw. mm ist. Aus den gegebenen Daten von Regnault berechnen wir den Wassergehalt in den $\mathrm{H}_{2} \mathrm{SO}_{4}$-Lösungen, welche dieselbe Dampfspannung besitzen (Rubrik 2 und 3 der Tabelle VI).

Berechnen wir aus der Tabelle V die Dampfspannung bei $30^{\circ}$ für Stärkearten, welche die in der Rubrik 2 angegebene Wassermenge enthalten, so erhalten wir die Werte der Rubrik 4, und berechnen wir nach den Daten von Regnauit die Dampfspannung bei $30^{\circ}$ der $\mathrm{H}_{2} \mathrm{SO}_{4}$-Lösungen, welche die in det Rubrik 3 angeführte Wassermenge enthalten, so erhalten wir dic Rubrik 5. Die einzelnen Werte in den Rubriken 4 und 5 kommen einander sehr nahe, der größte Unterschied ist bei geringem Druck, das teilweise dadurch zu erklären ist, dak hier die Kurven der Dampfspannung, sowohl der $\mathrm{H}_{2} \mathrm{SO}_{4}$-Lösungen als auch der Stärke, eine Knickung erleiden, und infolgedessen die Interpolation größere Fehler ergibt. Die Resultate der Berechnung bestätigen die angeführte Annahme, dab die Temperaturkoeffizienten der Dampfspannung des Wassers in den $\mathrm{H}_{2} \mathrm{SO}_{4}$ Lösungen und in der Stärke einander sehr nahe kommen. In dieser Approximation der erwähnten Koeffizienten haben wir die erste Analogie $z$ wischen der Wasserlösung in der Schwefelsaure und in der Stärke.

In der vorletzten Rubrik der Tabelle VI sind die Quotienten aus der Division der Zahlen der dritten Rubrik durch die Zahlen der zweiten Rubrik angeführt; diese Quotienten zeigen, wieviel Prozent Wasser in den $\mathrm{H}_{2} \mathrm{SO}_{4}$-Lösungen dieselbe Dampfspannung besitzen, wie ein Prozent Wasser in der bestimmten Stärke. Der charakteristische "Gang" der Zahlen der vorletzten Rubrik weist auf die Möglichkeit hin, eine Konstante zu erhalten, sobald wir von dem Dividendus eine gewisse Zahl subtrahieren. In der letzten Rubrik finden wir die Quotienten für den Fall, wenn man von den Zahlen der dritten Rubrik 15,4, d. h. die Zahl, die den Prozentgehalt des Wassers im Hydrat $\mathrm{H}_{2} \mathrm{SO}_{4} \cdot \mathrm{H}_{2} \mathrm{O}$ angibt, abzieht. Zieht man die Fehler des Versuchs und der Berechnung mit Hilfe der Interpolation in Betracht, so müssen wir die Werte der letzten Rubrik praktisch als konstant bezeichnen (die Schwankungen betragen 2,93 bis 2,52). Der ungezwungene ,Gang der Konstante" zu Anfang bängt aller Wahrscheinlichkeit nach von der Dissoziation des Hydrats $\mathrm{H}_{2} \mathrm{SO}_{4} \cdot \mathrm{H}_{2} \mathrm{O}$ ab. Die angeführten Berechnungen zeigen, dall die ersten 15 bis 16 Proz. (bei $19^{\circ}$ ) Wasser sich mit der Schwefelsäure mit einer Gewalt verbinden, die sich wesentlich von der unterscheidet, mit der die übrigen Wasserprozente sich verbinden; diese letzteren Prozente benehmen sich ebenso wie auch das Wasser in der Stärke, d. h. in den Adsorptionsverbindungen. Hier finden wir die $z$ weite Analogie $z$ wischen den Gelen und den gewöhnlichen Lösungen. Wenn eine funktioneile Abhängigkeit zwischen der Wassermenge in der Stärke und der Dampfspannung gefunden wäre, so müßte dieselbe Abhängigkeit auch für die $\mathrm{H}_{2} \mathrm{SO}_{4}$-Lösungen Geltung haben.

Zum Schluß halte ich es für meine Pflicht, hiermit dem Direktor des chemischen Laboratoriums des Finanzministeriums, Herrn Privatdozent A. Doroschewski, meinen Dank für die liebenswürdige Erlaubnis, die vorliegende Arbeit im genannten Laboratorium ausführen zu dürfen, auszusprechen.

Moskau, Mai 1911.

\title{
Studien über das Gleichgewicht im System $\mathrm{ZnSO}_{4}+\mathrm{KOH}+\mathrm{H}_{2} \mathrm{O}$.
}

\author{
Von S. Tschuman off.
}

(Eingegangen den 14. Oktober 1911)

(Aus dem Laboratorium der allgemeinen Chemie der Berg- und Hutten-Hochschule in Jekaterinoslaw.)

Es wurden acht Kolben yon $250 \mathrm{ccm}$ Inhalt, jeder mit $12 \mathrm{~cm}$ einer $\mathrm{ZnSO}_{4} \cdot 7 \mathrm{H}_{2} \mathrm{O}$-Lösung, die 0,00206 Mole Zinksulfat enthielt, versehen. Dann wurden die Kolben aus einer Bürette mit einer titrierten $\mathrm{KOH}$-Lösung in allmählich zunehmender Konzentration versetzt, bis zur
Marke mit destilliertem Wasser aufgefüllt und unter häufigem Schütteln während zehn Tagen stehen gelassen.

Parallel wurde eine Reihe derselben Versuche zur Kontrolle angesetzt. 